\title{
\%
}

\section{EL DESARROLLO DE LA ECONOMÍA COLABORATIVA Y LOS MODOS DIGITALES DE PRESTACIÓN DE SERVICIOS}

La economía colaborativa puede definirse como el aprovechamiento de recursos infrautilizados a través de la interacción en plataformas digitales. Este fenómeno está explicado por poderosas dinámicas económicas, tecnológicas, socioculturales e incluso regulatorias, cuestiones que deben ser tenidas en cuenta por los modelos de negocio que deseen prosperar en este ámbito. Los sectores donde la economía colaborativa se está desarrollando con más vigor son el transporte, el alojamiento, las finanzas y los servicios profesionales. Las autoridades públicas deben responder a este fenómeno con una regulación económica eficiente, pues tiene numerosas ventajas para la economía, entre ellas las competitividades interna y externa.

Palabras clave: economía colaborativa, economía digital, competencia, regulación económica eficiente, competitividad.

Clasificación JEL: K21, L40, L50, L51.

\section{Introducción}

La palabra «disrupción» es la que mejor puede resumir los cambios generados por la economía colaborativa y los nuevos modos digitales de prestación de servicios. Este componente disruptivo está planteando notables retos a la hora de adaptarse a este fenómeno, tanto

\footnotetext{
* Subdirectora de Estudios e Informes. Departamento de Promoción de la Competencia de la CNMC. Técnico Comercial y Economista del Estado.

** Vocal Asesor de la Subdirección de Estudios e Informes. Departamento de Promoción de la Competencia de la CNMC. Técnico Comercial y Economista del Estado.

Versión de abril de 2017.

Las opiniones expresadas en el presente artículo son de los autores. Los autores desean agradecer las aportaciones de Antonio Maudes Gutiérrez, Alfonso González Aparicio y Raquel Tárrega López. Los errores u omisiones que pudieran existir son en cualquier caso responsabilidad exclusiva de los autores.
}

para los negocios tradicionales ya establecidos como para las autoridades públicas. $Y$ estos retos se traducen en acalorados debates sobre cuál debe ser la respuesta regulatoria más adecuada para salvaguardar el bienestar general.

Estas controversias llegan incluso al plano teórico, pues también existe debate sobre cuál es la definición más atinada de economía colaborativa ${ }^{1}$. Es decir, la propia disrupción de la economía colaborativa es también $\triangleright$

\footnotetext{
1 En esta controversia quizás influye que el término inglés sharing economy tiene un matiz más de compartir (recursos infrautilizados) que de colaborar, con lo que la traducción que se ha generalizado en castellano de «economía colaborativa» puede alterar las connotaciones. En este sentido, algunos autores (Belk, 2014) prefieren distinguir el pseudo-sharing, que se realiza a cambio de una compensación, del true sharing.
} 
académica, pues es un fenómeno que ha llegado sin los libros de textos preparados y que está redefiniendo las fronteras de la empresa que consideraba el economista Ronald Coase (Munger, 2015).

Dada esta controversia sobre el concepto exacto de economía colaborativa, es preferible optar por una perspectiva amplia. Algunos autores (Codagnone et al., 2016) recomiendan realizar definiciones pragmáticas más basadas en ejemplos (ostensive) que en aquellas basadas en connotaciones (intensional). En este sentido podemos destacar ciertos elementos comunes a la economía colaborativa y a los nuevos modos digitales de prestación de servicios.

El primer aspecto que cabe destacar es el uso de recursos infrautilizados, ya sean bienes duraderos (como el vehículo propio) o activos (como la vivienda) en manos de hogares, activos productivos de las empresas (como espacio logístico) e incluso valores más intangibles como el tiempo o el capital humano.

Pero el aprovechamiento común de recursos infrautilizados no es exclusivo del momento actual, es tan antiguo como la propia humanidad (Codagnone y Martens, 2016). La verdadera novedad (y lo que genera disrupción en negocios establecidos y en reguladores) es la escala con la que este fenómeno se está produciendo actualmente, pasando de circunscribirse a círculos locales y transacciones anecdóticas entre seres muy próximos a ampliarse a la esfera global y permitiendo intercambios entre desconocidos. Para entender este desarrollo cabe citar una serie de elementos definidores de la actual ola de economía colaborativa en el siglo xxı, más allá del uso de recursos ociosos.

El primero de estos aspectos es el uso de tecnologías digitales para facilitar las transacciones, lo que reduce enormemente los costes de transacción y aumenta la eficiencia en el emparejamiento (matching). El segundo es la emergencia de la figura del «prosumidor», un ciudadano-productor que se puede situar a ambos lados del mercado: oferta y demanda.

$Y$ el tercero es el aprovechamiento de estructuras de plataforma (matchmaker) en mercados de múltiples lados (multi-sided markets) donde se producen los denominados efectos (o externalidades) de red indirectos(as): el valor de una plataforma para un lado (por ejemplo, el demandante) aumenta a medida que se incrementan los agentes en otro lado (oferentes). Esto puede llevar a dinámicas de concentración de oferta y demanda, pues si una plataforma tiene muchos agentes de un lado (oferentes), le será más fácil atraer al otro lado (demandantes), lo que a su vez vuelve a atraer más agentes del primer lado (oferentes), generando una retroalimentación positiva ${ }^{2}$. Para fortalecer estos efectos de red indirectos, las plataformas de economía colaborativa suelen añadir mecanismos de puntuación (rating) tanto para oferentes como para demandantes, de forma que se genere confianza a ambos lados del mercado y se puedan producir estas transacciones entre extraños.

Tras esta aclaración de lo que entendemos por economía colaborativa, el presente artículo tiene como objeto describir con detalle ciertos aspectos de su desarrollo. Por ello, tras este primer apartado introductorio, el segundo se adentra en los factores que explican el auge de la economía colaborativa, con ánimo de ser breve, pero preparando el terreno para $\triangleright$

2 Es fácil pensar en el juego de estos efectos en los dos principales sectores de la economía colaborativa (por este orden): transporte y turismo. Un consumidor valorará tener una oferta amplia de conductores y alojamientos y los oferentes valorarán una red amplia de usuarios, retroalimentándose ambos efectos a la hora de atraer agentes de ambos lados del mercado. 
comprender bien los dos siguientes apartados. El tercer apartado explica cuáles son las claves para el desarrollo de un modelo de negocio exitoso en el ámbito de la economía colaborativa, tratando de extraer lecciones transversales que se apliquen a todas las actividades. El cuarto apartado sí adopta una perspectiva sectorial para detallar en qué campos se ha desarrollado más la economía colaborativa y por qué. El quinto apartado abordará las conclusiones, con un debate sobre los efectos de la economía colaborativa a partir del cual se pueden establecer unas recomendaciones a los poderes públicos para que estos den una respuesta adecuada a este fenómeno.

\section{Factores de desarrollo de la economía colaborativa}

Sin ánimo de exhaustividad, los factores que explican el desarrollo de la economía colaborativa se pueden reunir en tres grandes grupos: económicos, tecnológicos y socioculturales.

Entre los factores económicos se ha tendido a destacar la situación de crisis, por dos vías. Por el lado de la oferta, los ciudadanos pueden actuar como agentes proveedores de servicios tratando de superar una pérdida de ingresos monetizando activos infrautilizados, como su vivienda, su vehículo y otros bienes duraderos o su tiempo libre combinado con su capital humano. Por el lado de la demanda, los ciudadanos, que han visto minorada su renta y dificultado su acceso al crédito, pueden satisfacer sus necesidades en términos de acceso a servicios en lugar de hacerlo sobre la tenencia de bienes en propiedad.

Pero, dado que la economía colaborativa sigue creciendo en lugares y contextos donde ya no se siente tanto la crisis, es evidente que subyacen otros factores económicos de índole estructural y no coyuntural. En este sentido destacan las enormes ganancias de eficiencia fruto de la reducción de costes de transacción, por la mayor facilidad para informarse sobre las posibilidades de realizar intercambios, negociar sobre las condiciones y casar (match) efectivamente oferta y demanda. Otra explicación de la mayor eficiencia de la economía colaborativa para permitir transacciones es la reducción de asimetrías informativas por las herramientas de calificación (rating) de oferentes y demandantes, que introducen mecanismos de reputación.

Estos factores económicos en forma de ganancias de eficiencia (reducción de costes de transacción y de asimetrías informativas) no se entienden sin introducir otros aspectos tecnológicos y sociales, igualmente estructurales. En el ámbito tecnológico destacan múltiples elementos: la mayor interactividad en internet, la conectividad por el internet móvil y la geolocalización por GPS, la revolución del ecosistema de aplicaciones móviles en «teléfonos inteligentes» (smartphones), etcétera. En el ámbito sociocultural, el cambio más relevante ${ }^{3}$ es la renuncia voluntaria al anonimato que asumen los individuos, con el principal exponente de las redes sociales.

La interacción de todos estos factores es lo que permite que la economía colaborativa (como aprovechamiento de recursos infrautilizados) se desarrolle al nivel que estamos observando. El desarrollo de intercambios a escala global no se puede entender sin la eficiencia económica y tecnológica de estas herramientas y plataformas digitales. Pero, $D$

3 Otro aspecto destacable son las preocupaciones medioambientales o la preferencia por un disfrute de bienes y servicios en términos de acceso y no de propiedad; sociocultural es la preferencia, especialmente por parte de consumidores jóvenes y urbanos (milennials). 
al mismo tiempo, la existencia de transacciones entre desconocidos no se puede desligar de los cambios culturales que han conducido a la explosión de las redes sociales y de la elaboración de perfiles en la red, pues ello es clave para aumentar la confianza y consolidar los mecanismos de reputación basados en las calificaciones de usuarios. $Y$ es que la extensión del volumen de intercambios es clave por los ya comentados efectos de red indirectos, que crean una retroalimentación positiva entre el crecimiento de ambos lados del mercado.

Aparte de los factores económicos, tecnológicos y socioculturales, hay autores (Mohammed, 2014; Edelman y Geradin, 2016) que enfatizan los aspectos regulatorios. Es decir, la economía colaborativa se ha desarrollado en sectores sometidos a una normativa profusa y, en muchos casos, desfasada. Esto atenaza el crecimiento de ciertos modelos de negocio y ha llevado al prosumidor a intentar organizar sus transacciones de una manera alternativa. Especialmente debido a que la economía colaborativa ha dado solución a ciertos fallos de mercado, como las asimetrías informativas, de manera más satisfactoria que la intervención normativa. No obstante, estas mismas autoridades reguladoras han tratado de responder también de una manera restrictiva hacia la economía colaborativa, aunque dejaremos para el final del artículo el debate sobre cuál debe ser la respuesta óptima de los poderes públicos.

Una vez vistos los factores que explican el desarrollo de la economía colaborativa a nivel agregado es más fácil entender la dinámica del crecimiento a nivel microeconómico de un modelo de negocio basado en economía colaborativa. A esto se dedica precisamente el siguiente apartado.

\section{Los modelos de negocio en economía colaborativa}

Como hemos comentado, uno de los aspectos inherentes a la economía colaborativa es la organización de las transacciones en plataformas digitales. Por ello, un modelo de negocio basado en la economía colaborativa tendrá que tener en cuenta los aspectos generales de los mercados de múltiples caras (multi-sided) o de plataformas. A ello se añadirán ciertos factores más específicos de la economía colaborativa, como el aprovechamiento de recursos infrautilizados o la importancia de la figura del prosumidor.

Comenzando con los aspectos generales de la economía de plataformas, hay que iniciar las reflexiones definiendo un mercado de múltiples lados como el espacio, físico o virtual, que permite la interacción de demandas de distintos grupos (Comisión Europea, 2016b). Las plataformas (matchmakers) son los agentes que operan como intermediarios entre ambos lados. Se diferencian de las empresas tradicionales, que se dedican a transformar inputs en outputs; de los revendedores (resellers), que compran y asumen la propiedad del bien para transferirlo a continuación mientras las plataformas sólo intermedian y la propiedad del bien pasa directamente del lado oferente al lado demandante; y de las empresas integradas verticalmente, que asumen no ya la propiedad del bien, sino la del agente de un determinado lado del mercado.

La definición de plataforma tampoco está exenta de controversia, aunque la literatura ha ido configurando varios elementos (Codagnone y Martens, 2016).

- Efectos de red indirectos: la interacción entre ambos lados del mercado presenta beneficios, al menos para uno de los $\triangleright$ 
grupos ${ }^{4}$. Es decir, existe interdependencia entre la demandas de ambos grupos.

- No neutralidad de precios: las externalidades de red provocan precisamente que la estructura de precios que decida la plataforma no es neutral. Es decir, la decisión de en qué medida se repercuten los costes a uno o a otro lado es clave para determinar el tamaño del mercado.

- Crecimiento no lineal: la retroalimentación positiva entre ambos lados del mercado provoca que el crecimiento del negocio se dispare a partir de una determinada masa crítica.

- Interacción directa: las plataformas permiten la interacción directa entre los lados del mercado, aunque pueden determinar condiciones de la transacción.

- Vinculación con la plataforma: los usuarios (a ambos lados del mercado) mantienen un perfil en la plataforma (platform affiliation), aunque ésta no asuma la propiedad de los activos y además permita el multi-homing (la posibilidad de que los agentes participen en otras plataformas).

Todos estos elementos deben ser tenidos en cuenta para que prospere un modelo de negocio basado en un mercado de plataforma, sobre todo a la hora de determinar la estructura de precios. La máxima de precio no inferior al coste de una empresa tradicional se altera (Evans y Schmalensee, 2015). En un entorno de externalidades de red indirectas, el precio se subvenciona a un lado del mercado (al lado más elástico al precio, normalmente al consumidor), incluso hasta niveles de precios nulos o

\footnotetext{
4 En general, la retroalimentación positiva entre ambos lados del mercado es bidireccional: los oferentes valoran que haya muchos demandantes y los demandantes valoran que haya muchos oferentes. Pero en algunos casos sólo hay efectos de red unidireccionales. Por ejemplo, en una red social los anunciantes prefieren que la red tenga muchos usuarios, pero estos pueden no estar satisfechos con que la red tenga muchos anunciantes.
}

negativos, cuando de hecho se facilitan al consumidor servicios adicionales. Esto permite atraer a muchos individuos de ese lado del mercado, lo que a su vez atrae muchos agentes del otro lado, que serán los que financien los costes de la plataforma. Por tanto, la estrategia de las plataformas ha de ser la de asegurar que se atrae a todos los lados del mercado (bring both sides on board), con un equilibrio adecuado entre usuarios y proveedores, para garantizar un mercado líquido.

La estrategia de precio inferior a coste también puede ser óptima desde un punto de vista intertemporal para intentar alcanzar una masa crítica que asegure la viabilidad de la plataforma. Estos mercados con efectos de red indirectos están sometidos a problemas de tipo «huevo y gallina» (chicken-and-egg) ${ }^{5}$, con lo que las plataformas con un reducido número de transacciones pueden no prosperar ante sus rivales más grandes y que llegaron primero (en el problema conocido como the winner takes it all). Una estrategia transitoria de subvencionar a ambos lados del mercado puede permitir alcanzar el punto de inflexión a partir del cual el negocio es rentable, momento en el que se pueden decidir las posibles fuentes de ingresos (servicios premium de pago, publicidad, monetización de los datos de los usuarios a través de su venta, etcétera).

Pero estas dinámicas de concentración de oferta y demanda asociadas a los efectos de red indirectos no implican necesariamente que las plataformas grandes y consolidadas no estén sometidas a competencia. Además de los instrumentos con los que cuentan las autoridades de competencia, en estos mercados tecnológicos se ha popularizado la expresión $\triangleright$

5 Una plataforma con una base baja de oferentes no atrae a suficientes demandantes, lo que a su vez desalienta a los oferentes. 
de que la «competencia está a un clic» (competition is a click away) para hacer patente la facilidad con la que el consumidor puede acceder a proveedores alternativos de un bien o servicio ${ }^{6}$. Adicionalmente, las plataformas más pequeñas podrían optar por estrategias de nicho y diferenciación, intentando especializarse en determinados segmentos de valor añadido. Por otro lado, aunque en muchos casos sea difícil competir en precio (si éste es nulo o negativo), las plataformas entrantes podrían competir en calidad (por ejemplo, con mayores estándares de privacidad o menores tiempos de espera). Por último, la competencia puede llegar no sólo por el lado de la demanda, sino también por el lado de la oferta, es decir, ofreciendo un servicio que a priori no parece competir con el de la plataforma original pero logra atraer la atención de los consumidores, que pueden desplazarse a la nueva plataforma.

Por todo ello, las plataformas, incluso aquéllas con una posición aparentemente muy consolidada, suelen invertir en calidad y diversificar su oferta para anticiparse a sus competidores actuales y potenciales. Para mantener y ampliar su base de usuarios, en lugar de imponer una exclusividad (single-homing) que desalentaría a los individuos (y les incentivaría a unirse a otras plataformas sin exclusividad), las plataformas tratan de crear ecosistemas que aseguren que los usuarios satisfacen la mayoría de sus necesidades con ellas mismas ${ }^{7}$.

Pero además de todos estos aspectos generales de los mercados de múltiples caras, las plataformas de economía colaborativa han de

6 De hecho, esta frase se ha quedado desfasada con las nuevas tecnologías (Maudes et al., 2016) y podría decirse que la competencia está ahora a un mero deslizamiento de dedo (competition is a swipe away).

7 Este sería el caso de una plataforma de alojamiento turístico que comienza a ofrecer servicios y experiencias más allá del hospedaje. cuidar ciertos factores específicos, especialmente la generación de confianza (trust) entre los distintos lados. Como tal, la confianza es relevante en prácticamente todas las plataformas, como una forma de atraer a ambos lados del mercado y así aprovechar plenamente los efectos de red indirectos. Pero la confianza es aún si cabe más decisiva en los modelos de economía colaborativa, por los dos rasgos específicos que hemos comentado al principio de este apartado: la monetización de activos infrautilizados y la emergencia de la figura del prosumidor.

El deseo de aprovechar mejor recursos infrautilizados supone que, en muchos exponentes de la economía colaborativa, el oferente desea seguir siendo el propietario del bien (por ejemplo, la vivienda o el vehículo) después de la transacción, que consiste en un alquiler o cesión temporal. Por ello, para que la transacción se complete, ambos lados tienen que tener confianza mutua (el demandante en la calidad del bien o servicio y el oferente en la buena conducta del usuario).

Por otro lado, la emergencia de la figura del prosumidor supone que muchos intercambios se producen entre iguales (P2P, peer to peer) en lugar de las tradicionales transacciones de empresa a consumidor (B2C, business to consumer). En principio, la ventaja de la empresa sobre el ciudadano-productor es que la primera puede invertir más en imagen de marca y generar una reputación que otorgue confianza al consumidor.

Por tanto, ¿cómo logran las plataformas de economía colaborativa que los individuos pongan en común sus recursos y cierren transacciones incluso entre individuos extraños? Fundamentalmente con sistemas de calificación (rating) bidireccional, donde cada parte puntúa al otro lado del mercado y así cada agente $\triangleright$ 
tiene una reputación como oferente y/o demandante, lo que cumple dos funciones. Por un lado, es un sistema de revelación de información que ayuda a la toma de decisiones óptimas. Por otro lado, incentiva a los agentes a una buena conducta, pues una buena reputación les permitirá celebrar más intercambios y en mejores condiciones.

El buen funcionamiento de los sistemas de reputación también permite aprovechar los efectos (externalidades) de red directos(as). En estos casos la utilidad de una plataforma para un lado (por ejemplo, el demandante) aumenta a medida que se incrementan los agentes en su mismo lado $^{8}$ (demandantes). Así, un consumidor valora más una red con muchos usuarios porque las calificaciones de los oferentes son más confiables para él, ya sea porque el número de evaluaciones es mayor (efecto cuantitativo) o porque aumenta la probabilidad de que sus conocidos (en cuyo criterio confía más) hayan efectuado evaluaciones (efecto cualitativo de agenda de contactos). Por ello, para mejorar la confianza en los sistemas de calificación, muchas plataformas exigen o incentivan la integración del perfil del usuario con las redes sociales.

Aparte de los mecanismos de evaluación y reputación, las plataformas ofrecen otra serie de atributos para mejorar la confianza. Entre ellos (Coyle, 2016) estarían el establecimiento de una plataforma online de interacción, la gestión de los sistemas de pago, la resolución de disputas, el servicio postventa, etcétera. De hecho, las plataformas también se convierten en autorreguladores privados de su propio mercado fijando ciertos estándares para el acceso y el ejercicio, aportando modelos de contrato y seguros, etcétera. Aunque sobre

\footnotetext{
8 Se aprecia, por tanto, la diferencia con los efectos indirectos, donde los agentes de un lado valoran el número de agentes en el lado contrario.
}

esta cuestión de la autorregulación entraremos al final del artículo, tras repasar en el próximo apartado en qué sectores se está desarrollando más la economía colaborativa y por qué.

\section{Los sectores más relevantes para la economía colaborativa}

Pese a la dificultad de obtener datos fiables, los análisis realizados (PwC, 2015, 2016 y 2017; Petropoulos, 2017; Rodríguez Marín, 2017) coinciden a grandes rasgos en destacar cinco áreas de crecimiento de la economía colaborativa. Se trata, por este orden, de transporte, turismo y alojamiento, sistema financiero, servicios profesionales y consumo personal (otros bienes y servicios a hogares). En este apartado vamos analizar brevemente las características de la economía colaborativa en cada uno de estos sectores, aunque antes cabe realizar un apunte transversal. En todos estos ámbitos de actividad se dan, en cierta medida, los elementos que hemos mencionado como específicos de la economía colaborativa, fundamentalmente la presencia de activos infrautilizados, la emergencia del prosumidor y la generación de eficiencias como la reducción de costes de transacción y la solución de asimetrías informativas.

- En relación con los recursos infrautilizados, es preciso recordar que hay que considerar tanto los bienes tangibles, como el vehículo en el caso del transporte y la vivienda en el caso del turismo, como el dinero para el ámbito de las finanzas, y valores más intangibles, como el tiempo libre y el capital humano para los servicios profesionales y a hogares.

- En lo que concierne al protagonismo del prosumidor, cabe destacar que ello ha $\triangleright$ 
posibilitado la entrada del individuo como agente productor en competencia con empresas en sectores que estaban altamente profesionalizados y regulados como el transporte, el alojamiento turístico, las finanzas y los servicios profesionales.

- En lo que respecta a la reducción de costes de transacción, el recurso a plataformas digitales posibilita mejores servicios de búsqueda, recomendación y formación de precios (basados en algoritmos) y una casación más eficiente de oferta y demanda. Esto ha transformado la realidad de sectores con una oferta dispersa, como el transporte, el alojamiento y los servicios profesionales.

- Finalmente, en relación con la corrección de asimetrías informativas, en muchos de estos sectores (transporte, turismo, servicios profesionales...) las interacciones tienden a ser no repetidas. Con la introducción de sistemas de reputación sí se pueden establecer interacciones repetidas (lo cual disciplina la buena conducta) y especialmente se establece un mecanismo de revelación de información que puede permitir confiar en la información general de la plataforma.

Comenzando ya con el análisis sectorial, el transporte es el sector que más volumen de recursos monetarios está generando (Petropoulos, 2017). Por el lado de la oferta, es evidente que esta viene inducida por la clara infrautilización del vehículo propio ${ }^{9}$. Por el lado de la demanda, es evidente que ésta está virando desde la necesidad de disponer de un vehículo privado hacia la satisfacción de

9 La mayoría de los vehículos privados están parados más de 23 horas al día y el escaso tiempo en que se usan suele ser por un único ocupante (OCDE, 2016). servicios de movilidad por múltiples vías, que no tienen por qué suponer el uso del propio automóvil (PwC, 2015). En la tendencia hacia la movilidad compartida también influyen las preocupaciones medioambientales (Hamari, Sjöklint, Ukkonen, 2013), y los cambios culturales (como la interacción entre desconocidos a través de herramientas como las redes sociales). Estos mayores volúmenes de oferta y demanda casan además mejor por factores tecnológicos (como la conectividad constante gracias al internet móvil y al GPS) y por la utilización de herramientas como el ajuste dinámico en precios.

Tras el transporte encontramos el sector turístico, especialmente el alojamiento, aunque también actividades conexas como la hostelería, el ocio y la cultura. En el caso del alojamiento, de nuevo la oferta está impulsada por un activo infrautilizado como es la vivienda. En el caso de los servicios conexos, también hay activos infrautilizados como la disposición de las personas a utilizar su tiempo y su conocimiento para proveer servicios gastronómicos, culturales, etcétera. Por el lado de la demanda, estas actividades como el turismo y la hostelería añaden a sus consumidores tradicionales los nuevos usuarios que quieren disfrutar de estos servicios de una manera innovadora (como el intercambio de casas, la sensación de disfrutar de experiencias de vida local, etcétera). En comparación con el transporte, que es una actividad relativamente estandarizable y en la que se valora mucho el precio y la conveniencia, los consumidores de servicios de turismo y áreas conexas tienen una preferencia por la variedad y la diferenciación, con mayor disposición a pagar por servicios de valor añadido.

El caso del sector financiero resulta especialmente interesante por dos temas que ya $\triangleright$ 
se han tratado a lo largo del artículo, como son el debate entre factores coyunturales y estructurales y el papel de la corrección de asimetrías informativas. En el desarrollo de las finanzas colaborativas ha podido también jugar un rol la crisis financiera, pues el canal bancario ha encarecido y racionado a los deudores el acceso a crédito mientras remuneraba a tipos muy bajos a los ahorradores. Por ello, ambos grupos de agentes (deudores y ahorradores) tenían incentivos a buscar alternativas para canalizar el ahorro hacia la inversión sin depender de la intermediación bancaria. Pero de nuevo, más allá de este factor coyuntural de la crisis, el auge de las finanzas colaborativas se explica por factores estructurales como la aplicación de nuevas tecnologías en este sector (FinTech). Y otro factor estructural es la mayor eficiencia de las plataformas, no sólo por la reducción de costes de transacción, sino especialmente por la corrección de asimetrías informativas gracias a los mecanismos de señalización y reputación, claves en un sector en el que estos problemas en la información son muy agudos. Todas estas ganancias permiten que la movilización de los particulares en la financiación participativa (crowdfunding) facilite a empresas (especialmente las pequeñas, nacientes e innovadoras, cuyo acceso al crédito bancario está más racionado) obtener recursos de forma menos onerosa de una base inversora más amplia.

El siguiente sector en llegar a la economía colaborativa es el de los servicios profesionales, con un gran potencial de futuro por la posibilidad de crear mecanismos de reputación e interacción repetida en un sector que también estaba sujeto a problemas de asimetrías informativas. Las plataformas de economía colaborativa también aportan un emparejamiento más eficiente y un acceso del usuario a una oferta más variada, en un ámbito donde la personalización y la especialización son especialmente valoradas por el consumidor.

Finalmente, el ámbito del consumo de los hogares abarca un capítulo muy amplio de sectores (PwC, 2016). Desde la compartición de bienes duraderos (también infrautilizados) hasta la compraventa de bienes de segunda mano (donde sí se transfiere la propiedad). También incluye servicios minoristas, como el reparto de bienes de consumo y alimentación, y servicios de compartición de contenido audiovisual, generalmente accesibles a demanda.

En definitiva, a lo largo del artículo hemos comprobado cómo los factores de desarrollo de la economía colaborativa son estructurales y cómo ésta se desarrolla de manera transversal (por sus mejoras de eficiencia) y especialmente en ciertos sectores (por sus características específicas). Ante estas dinámicas vamos a tratar de elaborar una serie de conclusiones, principalmente orientadas a formular recomendaciones a las Administraciones Públicas para que den una respuesta regulatoria apropiada.

\section{Conclusiones e implicaciones para las autoridades públicas}

La prestación de servicios de economía colaborativa a partir de plataformas digitales responde a factores estructurales que muestran que estamos ante un fenómeno imparable. Además, la economía colaborativa ofrece múltiples ventajas, comenzando por el empoderamiento del prosumidor, en sus dos facetas. Como consumidor, por el disfrute de una mayor oferta y mayor variedad a precios más eficientes, y por el acceso a mayor y mejor información. Como ciudadano-productor, por la reducción de las barreras de $\triangle$ 
acceso y el aprovechamiento de activos infrautilizados. Esto genera una mayor eficiencia a nivel agregado en toda la economía, aparte de otros efectos como la innovación (por el contenido tecnológico de estos servicios), la conservación del medio ambiente (por el uso de recursos ociosos) y la posible mejora de la distribución de la renta (por la monetización de activos de los hogares de renta media-baja).

La respuesta óptima de los poderes públicos ha de ser, por tanto, permitir el desarrollo de la economía colaborativa a pleno potencial, sin trabas que impidan materializar las ventajas que acabamos de mencionar. De existir intervención pública, ésta debe guiarse por los principios de regulación económica eficiente, destacando la necesidad (justificación por un fallo de mercado u otra razón imperiosa de interés general) y proporcionalidad (asegurando el mínimo perjuicio para la competencia).

Estos mismos principios han de aplicarse a la revisión de la normativa sectorial existente, pues ya hemos comentado que ésta es abundante en ciertos ámbitos de actividad donde los modelos de negocio tradicionales no han sabido adaptarse a las nuevas demandas de los consumidores tan rápido como lo han hecho las plataformas digitales de economía colaborativa.

Precisamente, la economía colaborativa ofrece ventajas para la intervención reguladora de las Administraciones Públicas en dos aspectos. Por un lado está la corrección de asimetrías informativas a través de los mecanismos de reputación, que informan al consumidor sobre la calidad e incentivan una conducta responsable de los agentes. Esto hace que cierta normativa que pretendía establecer requisitos de calidad quede desfasada, pudiendo suponer una liberación de recursos que hasta ahora se vienen destinando a la actividad regulatoria y supervisora.
Por otro lado está la operativa de los mercados de múltiples lados, donde la plataforma es la primera interesada en asegurar la calidad del servicio, pues un descenso de la misma expulsaría a agentes de un lado del mercado, lo que acabaría desalentando a los agentes del otro lado (por juego de los efectos de red indirectos). Por ello, las plataformas acaban estableciendo mecanismos de autorregulación privados de su propia actividad, estableciendo requisitos de acceso (si bien mínimos en comparación con la regulación pública) y ejercicio (con estándares, contratos, seguros, arbitraje e incluso con la eventual expulsión de la plataforma a agentes con bajas puntuaciones).

Aparte de la revisión de la regulación sectorial, los poderes públicos también tienen el desafío de adaptar su normativa horizontal, sobre todo en materia fiscal y laboral. La figura del prosumidor, que participa en ambos lados del mercado y realiza tareas en múltiples sectores, es una novedad que debe obligar a modernizar ciertas disposiciones legales. Pero de nuevo la economía colaborativa ofrece también ventajas para la supervisión en materia fiscal y laboral, pues la trazabilidad de las transacciones es mayor en las plataformas digitales que en la economía tradicional.

Por último, queremos acabar el artículo con una reflexión sobre la perspectiva exterior de la economía colaborativa. A priori podría pensarse que se trata de un fenómeno con una dimensión local y nacional porque la mayoría de actividades en las que se desarrolla son del sector servicios y son poco comerciables internacionalmente. Sin embargo, esta visión es errónea por varios motivos.

En primer lugar, los sectores no comerciables también son relevantes para la competitividad exterior de una economía. Una mayor eficiencia, productividad y competencia en $\triangleright$ 
la provisión de los mismos, como la que genera la economía colaborativa, puede liberar recursos que se pueden invertir en otras áreas como los sectores exportadores.

En segundo lugar, la economía colaborativa también se está desarrollando en sectores que sí son exportables. Entre ellos destaca el turismo, sector clave para España y en el que es clave tener una oferta diversificada para adaptarse a las cambiantes exigencias de los viajeros. Pero también hay otros sectores cada vez más exportables, como los servicios financieros y los servicios profesionales a empresas, áreas de alto valor añadido cuya diferenciación vuelve ser un vector de competitividad. $Y$ el transporte de pasajeros, aunque a priori no sea comerciable internacionalmente, es una actividad que en gran medida está ligada al turismo y es clave para la competitividad de una economía.

En este sentido cabe destacar dos documentos recientes de la Comisión Europea. El primero (Comisión Europea, 2016a) es una Comunicación sobre las plataformas online en que la Comisión insta a los Estados miembros al desarrollo de una buena regulación, bajo los principios de necesidad y proporcionalidad, para asegurar que no se impide la consolidación del Mercado Único Digital. El segundo documento (Comisión Europea, 2017) vincula la corrección y prevención de desequilibrios macroeconómicos con reformas a nivel microeconómico en varios ámbitos, destacando en múltiples ocasiones la necesidad de una regulación económica eficiente que permita materializar los efectos positivos de la economía colaborativa. Estos vínculos con la competitividad y la integración exterior son un motivo más para adoptar una respuesta de política económica decidida que permita el desarrollo a pleno potencial de la economía colaborativa.

\section{Bibliografía}

[1] BELK, R. (2014). "Sharing versus pseudosharing in web 2.0\%. Anthropologist, vol. 18, n. -1 , pp. 7-23.

[2] CNMC (2016). Conclusiones preliminares del Estudio de los nuevos modelos de prestación de servicios y la economía colaborativa.

[3] CODAGNONE, C. y MARTENS, B. (2016). «Scoping the Sharing Economy: Origins, Definitions, Impact and Regulatory Issues". JRC Technical Reports, Institute for Prospective Technological Studies, Digital Economy Working Paper 2016/01.

[4] CODAGNONE, C.; BIAGI, F. y ABADIE, F. (2016). «The Passions and the Interests: Unpacking the 'Sharing Economy'». JRC Science for Policy Report.

[5] COMISIÓN EUROPEA (2016a). Online Platforms and the Digital Single Market: Opportunities and Challenges for Europe.

[6] COMISIÓN EUROPEA (2016b). Staff working document accompanying the Communication on Online Platforms and the Digital Single Market.

[7] COMISIÓN EUROPEA (2017). Country Report Spain 2017. Including an In-Depth Review on the prevention and correction of macroeconomic imbalances.

[8] COYLE, D. (2016). «Making the most of platforms: a policy and research agenda». Toulouse School of Economics, octubre.

[9] EDELMAN, B. y GERADIN, D. (2016). «Spontaneous Deregulation». Harvard Business Review, abril.

[10] EVANS, D.S. y SCHMALENSEE (2015). «The Antitrust Analysis of Multi-Sided Platform Businesses». NBER Working Paper no. 18.783 , febrero.

[11] HAMARI, J.; SJÖKLINT, M. y UKKONEN, A. (2013). «The Sharing Economy: Why People Participate in Collaborative Consumption", Social Science Research Network, vol. 67, n.ำ 9 .

[12] MAUDES, A.; SOBRINO, M. y HINOJO, P. (2016). «The sharing economy and new models of service delivery". OECD insights, junio.

[13] MOHAMMED, R. (2014). «Regulation Is Hurting Cabs and Helping Uber». Harvard Business Review, julio. 
[14] MUNGER, M. (2015). "Coase and the 'sharing economy'». In VELJANOVSKI, C. Forever contemporary: the economics of Ronald Coase. London: The Institute of Economic Affairs in association with London Publishing Partnership Ltd.

[15] OCDE (2016). «Competition and innovation in land transport». DAF/COMP/WP2(2016)6, Working Party no. 2 on Competition and Regulation, Background Note by the Secretariat.

[16] PETROPOULOS, G. (2017). «An economic review of the collaborative economy». Bruegel

[17] PwC (2015). «The Sharing Economy». Consumer Intelligence Series.

[18] PwC (2016). Assessing the size and presence of the collaborative economy in Europe.

[19] PwC (2017). Sharing Economy 2017 Predicitions.

[20] RODRÍGUEZ MARÍN, S. (2017). Los modelos colaborativos y bajo demanda en plataformas digitales. Sharing España y Asociación Española de la Economía Digital. 